\title{
Title: Modification of Heterogeneous Chemistry by Complex Substrate Morphology
}

\author{
Author(s): $\quad$ B. F. Henson, CST-6 \\ S. J. Buelow, CST-6 \\ J. M. Robinson, CST-6
}

Submitted to:

DOE Office of Scientific and Technical Information

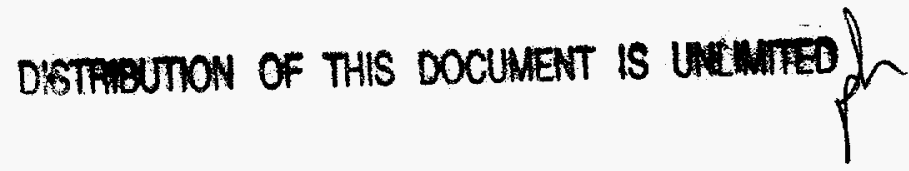

\section{Los Alamos}

NATIONAL LABORATORY

Los Alamos National Laboratory, an affirmative action/equal opportunity employer, is operated by the University of California for the U.S. Department of Energy under contract W-7405-ENG-36. By acceptance of this article, the publisher recognizes that the U.S. Government retains a nonexclusive, royaltyiree license to publish or reproduce the published form of this contribution, or to allow others to do so, for U.S. Government purposes. Los Alamos National Laboratory requests that the publisher identify this article as work periormed under the auspices of the U.S. Department of Energy. Los Alamos National Laboralory strongly supports academic freedom and a researcher's right to publish; as an institution, however, the Laboratory does not endorse the viempoint of a publication or guarantee its technical correctness. 


\section{DISCLAIMER}

This report was prepared as an account of work sponsored by an agency of the United States Government. Neither the United States Government nor any agency thereof, nor any of their employees, makes any warranty, express or implied, or assumes any legal liability or responsibility for the accuracy, completeness, or usefulness of any information, apparatus, product, or process disclosed, or represents that its use would not infringe privately owned rights. Reference herein to any specific commercial product, process, or service by trade name, trademark, manufacturer, or otherwise does not necessarily constitute or imply its endorsement, recommendation, or favoring by the United States Government or any agency thereof. The views and opinions of authors expressed herein do not necessarily state or reflect those of the United States Government or any agency thereof. 


\section{DISCLAMVIER}

Portions of this document migy be illegible in electronic image produets. Impges are produced from the best arailable original docmonent 


\title{
Modification of Heterogeneous Chemistry by Complex Substrate Morphology
}

\author{
Bryan F. Henson ${ }^{*}$, Steven J. Buelow, and Jeanne M. Robinson \\ Chemical Science and Technology Division
}

\begin{abstract}
This is the final report of a one-year, Laboratory Directed Research and Development (LDRD) project at Los Alamos National Laboratory (LANL). Chemistry in many environmental systems is determined at some stage by heterogeneous reaction with a surface. Typically the surface exists as a dispersion or matrix of particulate matter or pores, and a determination of the heterogeneous chemistry of the system must address the extent to which the complexity of the environmental surface affects the reaction rates. Reactions that are of current interest are the series of chlorine nitrate reactions important in polar ozone depletion. We have applied surface spectroscopic techniques developed at LANL to address the chemistry of chlorine nitrate reactions on porous nitric and sulfuric acid ice surfaces as a model study of the measurement of complex, heterogeneous reaction rates. The result of the study is an experimental determination of the surface coverage of one adsorbed reagent and a mechanism of reactivity based on the dependence of this coverage on temperature and vapor pressure. The resulting mechanism allows the first comprehensive modeling of chlorine nitrate reaction probability data from several laboratories.
\end{abstract}

\section{Background and Research Objectives}

Chemistry in many environmental systems is determined at some stage by heterogeneous reaction with a surface. Some examples include the reaction of urban pollutants with carbonaceous soots or aerosol fogs, reactivity and transport of contaminants in soils, the chemistry of combustion products on ash in industrial incineration, and most recently, the destruction of stratospheric ozone on cloud particulate. In all of these systems the strategy of understanding the heterögeneous chemistry involves simulating the surface in the laboratory and applying well-known techniques of surface chemistry applicable to tlat surfaces, or gas phase chemistry to indirectly probe the destruction of reagents and formation of products. Typically, however, the relevant surface exists as a dispersion or matrix of particulate matter or pores, and the molecular surface is subject to severe perturbations as a result of the extreme macroscopic curvature induced by diameters on the order of $10 \AA$ to $1000 \AA$. This is true of all the examples presented above. In addition, other mechanisms, such as

\footnotetext{
*Principal Investigator, E-mail: henson@lanl.gov
} 
the constraint of transport properties, reflected in increased collision frequencies with confining walls and the restriction of mixing in a porous sample also may affect the chemistry of the surface-reactant system. A true determination of the heterogeneous chemistry of a particular system must therefore address the extent to which the complexity of the environmental surface affects reaction rates determined on an ideal, infinitely flat surface.

The most severe perturbation induced by extreme curvature is through the surface chemical potential. Described by the Kelvin effect, ${ }^{1}$ strong curvature induces significant changes in the thermodynamic properties of the interface, changing the equilibrium vapor pressure over a surface by as much as $70 \%$. This dramatic change in stability can lead to changes in the surface concentration of an adsorbate, or the equilibrium thermodynamic phase of a substrate. These thermodynamic perturbations can strongly alter surface meditated chemistry. Two major mechanisms characterize reaction at a flat surface. ${ }^{2}$ In the Langmuir-Hinshelwood (LH) mechanism, reaction occurs between two adsorbed molecules. The limiting rate as a function of concentration goes through a maximum and decreases to zero. In the Langmuir-Rideal (LR) mechanism, the reaction occurs between an adsorbed species upon collision with a gas phase reactant. The limiting concentrationdependent rate in the LR mechanism is nonzero. These two mechanisms thus display dramatically different limiting rates as a function of surface concentration. In addition, these simple limits are valid for desorbing products that do not interfere with reaction.

Adsorbed products will inhibit the rate in a way that is strongly affected by the mobility of the adsorbed phase, typically saturating for a stationary phase and linearly increasing for a mobile phase. ${ }^{2}$ Modification of the kinetics of reaction on porous or particulate surfaces is thus possible either through the surface concentration or through the thermodynamic phase of a reagent or product. For molecular substrates, such as ice or glass phases, modification by the curvature can also proceed from the substrate itself. Extreme curvature must induce considerable surface heterogeneity due to the strain of projecting a rigid single crystal structure onto a curved topology. This heterogeneity may be expected to increase adsorption, given the strong interaction of adsorbed species with steps that is observed on metal and oxide surfaces.

Coupled reactions that are of considerable current interest and are susceptible to the type of modification discussed above include the series of chlorine nitrate reactions important in polar ozone depletion:

$$
\mathrm{ClONO}_{2}+\mathrm{HCl} \rightarrow \mathrm{Cl}_{2}+\mathrm{HNO}_{3}
$$




$$
\begin{array}{lll}
\mathrm{ClONO}_{2}+\mathrm{H}_{2} \mathrm{O} & \rightarrow \mathrm{HOCl}+\mathrm{HNO}_{3} \\
\mathrm{HOCl}+\mathrm{HCl} & \rightarrow \mathrm{Cl}_{2}+\mathrm{H}_{2} \mathrm{O}
\end{array}
$$

These reactions proceed on particulate ice in the stratosphere and serve to activate the $\mathrm{Cl}_{2}$ as a gas phase product, which subsequently attacks ozone. The history of the study of these reactions affords a lesson in the changing paradigm of investigations in heterogeneous chemistry in environmental systems.

The experimental investigation of these heterogeneous reactions and their impact on ozone depletion has developed in fairly well-defined stages. Initially the challenge was adequately simulating the low partial pressures and temperatures characteristic of the stratosphere. A considerable body of data characterizing the overall reaction rates under relevant conditions was quickly obtained and refined by measurements of the disappearance of reagent and appearance of products in the gas phase. ${ }^{3}$ As substrate ices reflecting the complexity of the stratospheric environment were addressed experimentally, it became apparent that simple rates obtained over a few surfaces would be inadequate to provide realistic predictive power in the complex environment of the stratosphere. For instance the rates of reactions 1 and $2 \mathrm{a}$ exhibit a dramatic dependence on the thermodynamic phase of a sulfuric acid aerosol, and due to extreme supercooling of the highly viscous liquid the phase (liquid, glass, crystallite) is a complicated function of the ambient conditions. It was quickly understood that a mechanistic understanding of these reactions was required so that a minimal set of parameters could be used to define the reactivity over the diversity of meteorology characteristic of the upper troposphere and lower stratosphere. Additional difficulties began to emerge due to the morphological complexities of the substrate itself. Major controversies continue in the literature. One concerns the extent to which the internal area of a porous, laboratory grown ice participates in the heterogeneous chemistry. ${ }^{3}$

The paradigm involves addressing successive stages of complexity. In the $1970 \mathrm{~s}$, upon consideration of chlorofluorocarbon (CFC) refrigeration technologies, calculations based entirely on homogeneous gas phase reactivity were performed to predict the impact of increased chlorine concentrations in the stratosphere. It was determined that chlorine would be removed in the form of stable compounds such as $\mathrm{ClONO}_{2}$ and $\mathrm{HOCl}$ and the impact on ozone would be minimal. ${ }^{4}$ With the discovery of the polar ozone hole it was realized that particulate matter in the stratosphere was reactivating chlorine through, among others, the heterogeneous mechanisms 1 and $2 .^{5}$ Since incorporation of the first few heterogeneous rate constants into models of the stratosphere, it has become apparent that full reaction mechanisms are required to define a sufficiently broad range of 
conditions, and that now the need is for a mechanistic understanding of the chemistry as a function of the full morphological and chemical complexity of the ice substrates.

We have demonstrated the ability to determine the surface localized concentration of several species of interest in the mechanism of 1 and 2. By the application of a surface sensitive spectroscopy, second harmonic generation ( $\mathrm{SHG}$ ), we have succeeded in monitoring the surface thermodynamic phase of $\mathrm{HNO}_{3} / \mathrm{H}_{2} \mathrm{O}$ ice. ${ }^{6}$ We have observed the condensation of $\mathrm{H}_{2} \mathrm{O}$ onto $\mathrm{HNO}_{3}\left(\mathrm{H}_{2} \mathrm{O}\right)_{3}$ acid hydrate substrates. We have also observed the adsorption of $\mathrm{HCl}$ onto $\mathrm{H}_{2} \mathrm{O}$ and $\mathrm{HNO}_{3}\left(\mathrm{H}_{2} \mathrm{O}\right)_{3}$ ice. The quantitative nature of the adsorption measurements derives from the ability to distinguish between the ice substrate and the adsorbate, the independent measurement of ice film surface area by physical adsorption, and the quantitative introduction of the adsorbed species as vapor over the ice.

We have developed a model of surface coverage to analyze the adsorption spectra obtained from the second harmonic data, which allow the determination of the mean adsorption energy in both the $\mathrm{H}_{2} \mathrm{O}$ on $\mathrm{HNO}_{3}\left(\mathrm{H}_{2} \mathrm{O}\right)_{3}$ and $\mathrm{HCl}$ on $\mathrm{H}_{2} \mathrm{O}$ ice systems. With these tools we have addressed the heterogeneity of the surface molecular potential. We have modeled the considerable data in the literature concerning the reaction probabilities of the heterogeneous reactions 1 and 2 utilizing surface reagent concentrations characterized by surface potentials measured in our laboratory.

\section{Importance to LANL's Science and Technology Base and National R\&D Needs}

Prior to the discovery of the so-called "ozone hole," heterogeneous processes were ignored in atmospheric chemistry models. Field measurements, supported by laboratory experiments and modeling, have confirmed the pivotal role of reactions of photoinactive (reservoir) chlorine and nitrogen gases on ice and nitric and sulfuric acid ice aerosols in the Antarctic polar vortex. It was subsequently recognized that the determination of chemical mechanism would be required in order to adequately model the heterogeneous rates as a function of the complex ice surfaces. Attention is now turning to mechanistic studies that incorporate the full thermodynamic and substrate complexity in these systems.

This work builds on a program unique to LANL with the capability to address these questions by probing the complex surface directly. The development of quantitative models of reactivity, which accurately model a wide range of reaction rate data, demonstrates the ability to deconvolute and characterize very complex and 
important heterogeneous chemical systems. This capability is one of the components of the new Atmospheric Sciences Initiative at LANL.

\section{Scientific Approach and Accomplishments}

The experimental approach has been built on a technique recently developed at the Laboratory ${ }^{6}$ to probe surface chemical composition directly utilizing SHG, a nonlinear surface spectroscopic technique. ${ }^{7}$ In general, the application of a high-power laser pulse to a surface results in the conversion of some of the light reflected from the surface to a frequency double that of the input pulse. The efficiency of this conversion process is a function of the chemical composition and thermodynamic phase of the surface. The generation process is also localized to the surface so that the technique represents a direct probe of the surface chemical composition and thermodynamic phase.

We have developed an application of this general technique to probe the molecular surface of transparent, porous ice films in order to characterize reagent concentration and coverage in reactions 1 and 2 . We use the $532 \mathrm{~nm}, 100 \mu \mathrm{J}$ pulse from a neodymium-doped yttrium-aluminum-garnet (Nd:YAG) laser for scattering from the surface of a porous ice film. We then apply the laser to the sample ice and obtain the doubled-frequency, second-harmonic-generation signal with a solar blind phototube and monochromater detection apparatus. We then introduce the adsorbate gas and monitor the change in second-harmonic generation as a function of surface concentration. Adsorption spectra are shown in Fig. 1 for the adsorption of $\mathrm{H}_{2} \mathrm{O}$ on $\mathrm{HNO}_{3}\left(\mathrm{H}_{2} \mathrm{O}\right)_{3}$. The adsorbed $\mathrm{H}_{2} \mathrm{O}$ serves to mask the second harmonic generation of the underlying substrate and the loss of signal observed as a function of the $\mathrm{H}_{2} \mathrm{O}$ concentration is a measure of the amount of the substrate covered.

The dependence of the second harmonic intensity on surface concentration can be expressed as

$$
I_{z \omega}=(\chi \theta)^{2}
$$

where $\chi$ is an average second harmonic efficiency and $\theta$ is a surface concentration. We apply the Brunauer, Emmett, and Teller (BET) physical adsorption theory in order to model the $\theta$ dependence of the SHG signal. ${ }^{8}$ From BET theory the total amount of adsorbate on the surface, $\theta$, is given by Equation 2 and the fraction of the total surface area not covered by adsorbate, $\theta_{\omega}$, by Equation 3 , both in units of monolayers.

$$
\theta=\left(\frac{\mathrm{cx}}{(1-\mathrm{x})[1+(\mathrm{c}-1) \mathrm{x}]}\right)
$$




$$
\theta_{0}=\left(1+\frac{c x}{(1-x)}\right)^{-1}
$$

The parameter $c \sim \exp \left(\left(Q_{s}-Q_{a}\right) / R T\right)$ depends on the relative adsorbate surface energy $Q_{s}$ and adsorbate-adsorbate energy $\mathrm{Q}_{\mathrm{a}}$. $\mathrm{R}$ is the gas constant and $\mathrm{x}=\mathrm{P} / \mathrm{P}_{\mathrm{o}}$ is the ratio of the vapor pressure to the pressure over the pure phase. The system is fully parameterized by the constant c, relating the amount of material adsorbed, the fractional surface coverage and the pressure.

One interpretation of adsorption data such as shown in Fig. 1 is that adsorption proceeds via multilayer formation, and that $\sqrt{\mathrm{I}_{2} \omega}$ is proportional to the fractional amount of substrate covered by adsorbate, irrespective of the depth of coverage, such that $\sqrt{I_{2} \omega} \propto$ $\left(1-\theta_{0}\right)$. Given this interpretation, the isotherms shown as $\sqrt{I_{2} \omega}$ versus coverage in Fig. 1 are equivalent to plotting $\left(1-\theta_{0}\right)$ against $\theta$. By combining equations 2 and 3 and eliminating $\mathbf{x}$, the $\mathrm{c}$ constant characterizing the adsorption system within the BET formalism may be directly determined from the spectra. This was the procedure for the calculation of the solid curves of Fig. 1. The shape of the curve is determined entirely by the $c$ constant, with the scattering efficiency, $\chi$, a linear scaling factor used to normalize the signal to one at zero coverage. The value of the constant, determined by least squares minimization, was 0.006 for $\mathrm{H}_{2} \mathrm{O}$ adsorption onto polycrystalline $\mathrm{HNO}_{3}\left(\mathrm{H}_{2} \mathrm{O}\right)_{3}$ at $190 \mathrm{~K}$ and 0.048 for adsorption at $85 \mathrm{~K}$.

In addition to modeling the adsorption spectra, we have used physical adsorption models to analyze data in the literature from other laboratories concerning the probabilities of reactions 1 and 2 under stratospheric conditions as a function of relative humidity. This model has been described fully in the literature. ${ }^{9}$ Briefly, the mechanism of reactivity in reactions 1 and 2 is considered to be prompt reaction between gas phase $\mathrm{ClONO}_{2}$ and adsorbed $\mathrm{H}_{2} \mathrm{O}$ or $\mathrm{HCl}$ upon collision at the surface, a classic example of the Langmuir-Rideal mechanism. ${ }^{2}$ The collision-based probability of reaction, $\gamma$, is the parameter typically measured in laboratory studies of reaction 1 and 2 . This $\gamma$ reflects the probability that a reaction will occur upon the collision with the surface by $\mathrm{ClONO}_{2}$. In the context of our collision mechanism, $\gamma$ is a simple function of the geometric coverage of the surface by either reagent $\mathrm{H}_{2} \mathrm{O}$ or $\mathrm{Hcl}$. This probability may be calculated for $\mathrm{ClONO}_{2}+\mathrm{H}_{2} \mathrm{O}$ as

$$
\gamma=\gamma_{0} \theta_{\mathrm{H}_{2} \mathrm{O}}=\gamma_{0}\left(1-\theta_{0}(\mathrm{c}, \mathrm{x})\right)
$$

where $\gamma_{0}$ is the reaction probability on a pure $\mathrm{H}_{2} \mathrm{O}$ ice. $\mathrm{HCl}$ coverage must be calculated on both the $\mathrm{H}_{2} \mathrm{O}$ ice and acid hydrate substrate domains for $\mathrm{ClONO}_{2}+\mathrm{HCl}$. In modeling these measurements we follow Tabazadeh and $\mathrm{Turco}^{10}$ and $\mathrm{Mozurkewich}^{11}$ and treat $\mathrm{HCl}$ 
adsorption as a single monolayer process via the Langmuir theory. The probability is then given by

$$
\gamma=\gamma_{0} \theta_{\mathrm{HCl}}=\gamma_{0}\left[\frac{\theta_{\mathrm{O}}(\mathrm{c}, \mathrm{x}) \mathrm{K}_{\mathrm{ACID}} \mathrm{P}_{\mathrm{HCl}}}{1+\mathrm{K}_{\mathrm{ACID}} \mathrm{P}_{\mathrm{HCl}}}+\frac{\left(1-\theta_{\mathrm{O}}(\mathrm{c}, \mathbf{x})\right) \sqrt{\mathrm{K}_{\mathrm{ICE}} \mathrm{P}_{\mathrm{HCl}}}}{1+\sqrt{\mathrm{K}_{\mathrm{ICE}} \mathrm{P}_{\mathrm{HCl}}}}\right]
$$

where $\theta_{\mathrm{HC}}$ is the coverage of $\mathrm{HCl}, \mathrm{K}_{\mathrm{ACID}}$ is the Langmuir adsorption constant for $\mathrm{HCl}$ on the acid hydrate ice, $\mathrm{K}_{\mathrm{ICE}}$ is the Langmuir adsorption constant for $\mathrm{HCl}$ on $\mathrm{H}_{2} \mathrm{O}$ ice, and $\mathrm{P}_{\mathrm{HCl}}$ is the $\mathrm{HCl}$ pressure. The adsorption of $\mathrm{HCl}$ is treated as dissociative on $\mathrm{H}_{2} \mathrm{O}$ ice and molecular on the acid hydrates, which is the reason for the square root dependence in the second term of Eq. 5. The coverage of the surface as a function of relative humidity is determined by the $\mathrm{c}$ constant, which we have measured in our laboratory from spectra such as Fig. 1 for the nitric acid trihydrate (NAT).

We show a comparison of calculations based on these measurements with measured reaction probabilities from other laboratories below. We also show comparison of calculations with data for probabilities over sulfuric acid tetrahydrate ice surfaces (SAT). For these calculations we have fit the $\mathrm{c}$ constant for the SAT surface, and derived a value of $c=0.2$ at $190 \mathrm{~K}$. The data ${ }^{9}$ and model calculation for $\gamma$ as a function of fractional relative humidity for $\mathrm{ClONO}_{2}+\mathrm{H}_{2} \mathrm{O}$ on both sulfuric acid tetrahydrate (SAT, filled data points) and nitric acid trihydrate (NAT, open data points) are shown in Fig. 2. Data for $\mathrm{ClONO}_{2}+\mathrm{HCl}$ are shown in Fig. 3. It is clear that this simple model successfully resolves at least two issues of concern regarding these data that have been expressed in the literature. The difference in reactivity between nitric and sulfuric acid ice substrates is driven by the different adsorption energy of $\mathrm{H}_{2} \mathrm{O}$ on each surface. This is indicated by the different $\mathrm{c}$ constant for NAT and SAT. The highly nonlinear behavior of reactivity with relative humidity is also resolved as a natural function of the $\mathrm{H}_{2} \mathrm{O}$ surface coverage with partial pressure.

The exception to the agreement of the physical adsorption model with the data is for $\mathrm{ClONO}_{2}+\mathrm{HCl}$ on a NAT substrate. The form of the curve for the probability of this reaction is commonly encountered in physical adsorption experiments involving porous media with pore radii in the $10 \AA$ to $1000 \AA$ range and is characterized as a type IV mesoporous isotherm. ${ }^{12}$ The curve reflects enhanced uptake by a porous solid induced by 
capillary absorption of liquid solution into the smallest pores. We also propose a reaction mechanism involving reaction in this capillary solution. The data for reaction with $\mathrm{HCl}$ are again plotted in Fig. 4. The labeled curve is calculated by reformulating the Kelvin equation as a function of $x$ as

$$
\mathrm{V}_{\mathrm{HCl}} \sim \mathrm{K}\left(\frac{\mathrm{dV}}{\mathrm{dx}}\right)\left(\frac{1}{\ln (\mathrm{x})}\right)
$$

where $\mathrm{V}_{\mathrm{HCl}}$ is the volume of liquid absorbed at $\mathrm{x}, \mathrm{K}$ is a constant proportional to the surface tension and molar volume of the solution, and $\mathrm{dV} / \mathrm{dx}$ is a Gaussian function describing the pore distribution in units of volume absorbed at $\mathrm{x}$. The reactivity is then modeled as

$$
\gamma=\gamma_{0} \mathrm{~V}_{\mathrm{HCl}} .
$$

In this model increased reactivity with relative humidity arises due to increasing liquid volume as condensation occurs in successively larger pores. We have not yet calculated the absolute volume of liquid involved, which requires the surface tension of the acidic solutions formed on condensation, and the solubility of $\mathrm{HCl}$ in such solutions.

The implication of this result is that the reported reaction probability for chlorine nitrate with $\mathrm{HCl}$ is actually a measure of reactivity in solution, not a measure of reaction mediated by the surface, and if the physical adsorption model presented here is indicative of the stratospheric process, then the uptake represented by the data for $\mathrm{ClONO}_{2}+\mathrm{HCl}$ on NAT may be too large by as much as an order of magnitude.

This last example of interference due to porosity is a prime illustration of the possible difficulties in interpretation of heterogeneous processes that may arise as a function of the complexity of the natural substrate, and highlights the goals of this project. We have shown that by directly measuring surface composition and thermodynamic phase, and by incorporating quantifiably complex features in the surface, such as porosity of known volume and area, it is possible to characterize very complex reaction systems. 


\section{Publications}

1. Henson, B. F., K. R. Wilson and Jeanne M. Robinson, "A Physical Adsorption Model of the Dependence of $\mathrm{ClONO}_{2}$ Heterogeneous Reactions on Relative Humidity," Geophys. Res. Lett., 23, 1021 (1996).

2. Henson, B. F., K. R. Wilson and Jeanne M. Robinson, "Modeling the Relative Humidity Dependence of Heterogeneous Reactions on Simulated PSC Surfaces," Poster presented at the 1995 fall meeting of the American Geophysical Union.

3. Henson, B. F., K. R. Wilson and Jeanne M. Robinson, "Chlorine Nitrate Reactivity on Simulated Polar Stratospheric Cloud Surfaces," Talk presented at the 211th American Chemical Society Meeting, March 1996.

4. Henson, B. F., K. R. Wilson and Jeanne M. Robinson, "Modeling the Relative Humidity Dependence of Chlorine Nitrate Reactivity on Ices," Talk presented at the 1996 International Symposium of the Physics and Chemistry of Ice, August 1996.

5. Henson, B. F., K. R. Wilson and Jeanne M. Robinson, "Laboratory Measurements of Heterogeneous Atmospheric Chemistry," Talk presented at the workshop, New Research in the Science of Global Change: A Multidisciplinary View, sponsored by the DOE, Santa Fe, NM, November 1995. 


\section{References}

1. Gregg, S. J. and K. S. W. Sing, Adsorption, Surface Area and Porosity, (Academic, 1982).

2. K. J. Laidler, Chemical Kinetics, (Harper \& Row, New York, 1987).

3. Kolb, C. E., D. R. Worsnop, M. S. Zahniser, P. Davidovits, L. F. Keyser, M.-T. Leu, M. J. Molina, D. R. Hanson, A. R. Ravishankara, L. R. Williams and M. A. Tolbert, "Progress and Problems in Atmospheric Chemistry," J. R. Barker, ed., in Adv. Series Phys. Chem., 3, C.-Y. Ng, ed., (World Scientific Publishing Co., 1995).

4. Kinnison, D., H. Johnston and D. J. Wuebbles, "Sensitivity Study of Global Ozone on NO Emission from Aircraft," in Proceedings of the international quadrennial ozone symposium, Gottingen, F. R. Germany, 8 August, 1988, UCRL-99361m, 1989; L. R. Martin, H. S. Judeikis, M. Wun, "Heterogeneous Reactions of Cl and ClO in the Stratosphere," J. Geophys. Res. Oceans 18, 5511 (1980).

5. Rowland, F. S, and M. J. Molina, "Chlorofluoromethanes in the Environment," Rev. Geophys. Space. Phys. 13, 1 (1975); Solomon, S. "Progress Towards a Quantitative Understanding of Antarctic Ozone Depletion," Nature 347, 347 (1990).

6. B. F. Henson, and J. M Robinson, "Surface Probes of Toluene/Water Ices: Adsorption Kinetics and Annealing Spectra Measured by Second Harmonic Generation," SPIE Proceedings, Laser Techniques for Surface Science, 2125, 140 (1994).

7. Shen, Y. R., The Principles of Nonlinear Optics (Wiley-Interscience, Toronto, 1984).

8. Henson, B. F., K. R. Wilson and Jeanne M. Robinson, "Quantitative Measurements of Multilayer Physical Adsorption on Heterogeneous Surfaces from Nonlinear Light Scattering," Phys. Rev. Lett., to be submitted.

9. Henson. B. F., K. R. Wilson and J. M. Robinson, "A Physical Adsorption Model of the Dependence of $\mathrm{ClONO}_{2}$ Heterogeneous Reactions on Relative Humidity," Geophys. Res. Lett. 23, 1021 (1996).

10. Tabazadeh, A. and R. P. Turco, "A Model for Heterogeneous Chemical Processes on the Surfaces of Ice and Nitric Acid Trihydrate Particles," J. Geophys. Res. 98, 12,727 (1993).

11. Mozurkewich, M., "Effect of Competitive Adsorption on Polar Stratospheric Cloud Reactions," Geophys. Res. Letters 20, 355 (1993).

12. Adamson, A. W., The Physical Chemistry of Surfaces (Wiley, New York, 1990). 


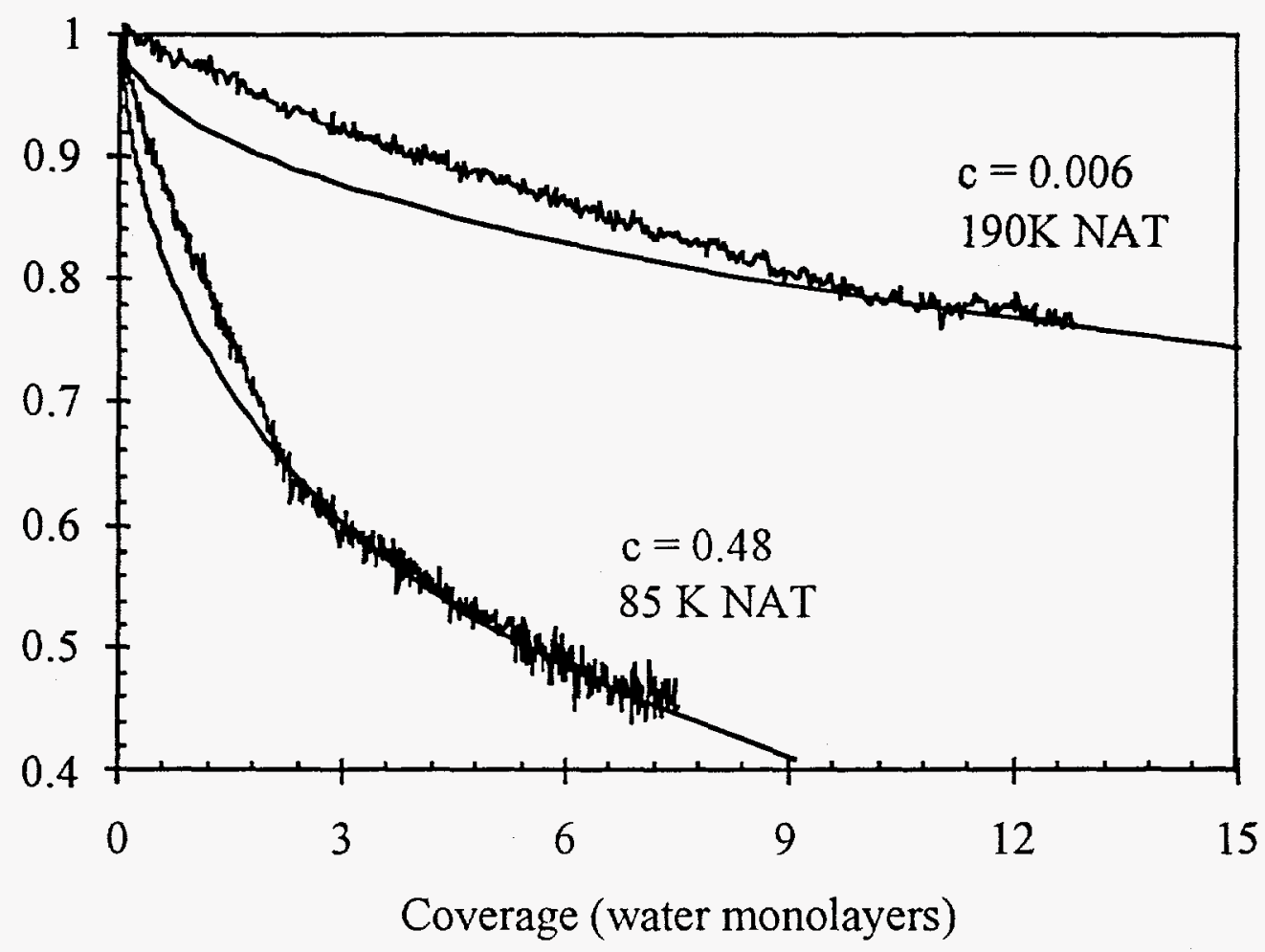

Figure 1. Coverage of porous NAT ice film by $\mathrm{H}_{2} \mathrm{O}$ at $85 \mathrm{~K}$ and $190 \mathrm{~K}$. The data are plotted as the square root of the second harmonic signal against $\mathrm{H}_{2} \mathrm{O}$ coverage. The solid curves are fit to the coverage model described in the text. The initial linear dependence arises due to conversion of a monohydrate surface layer to trihydrate. 


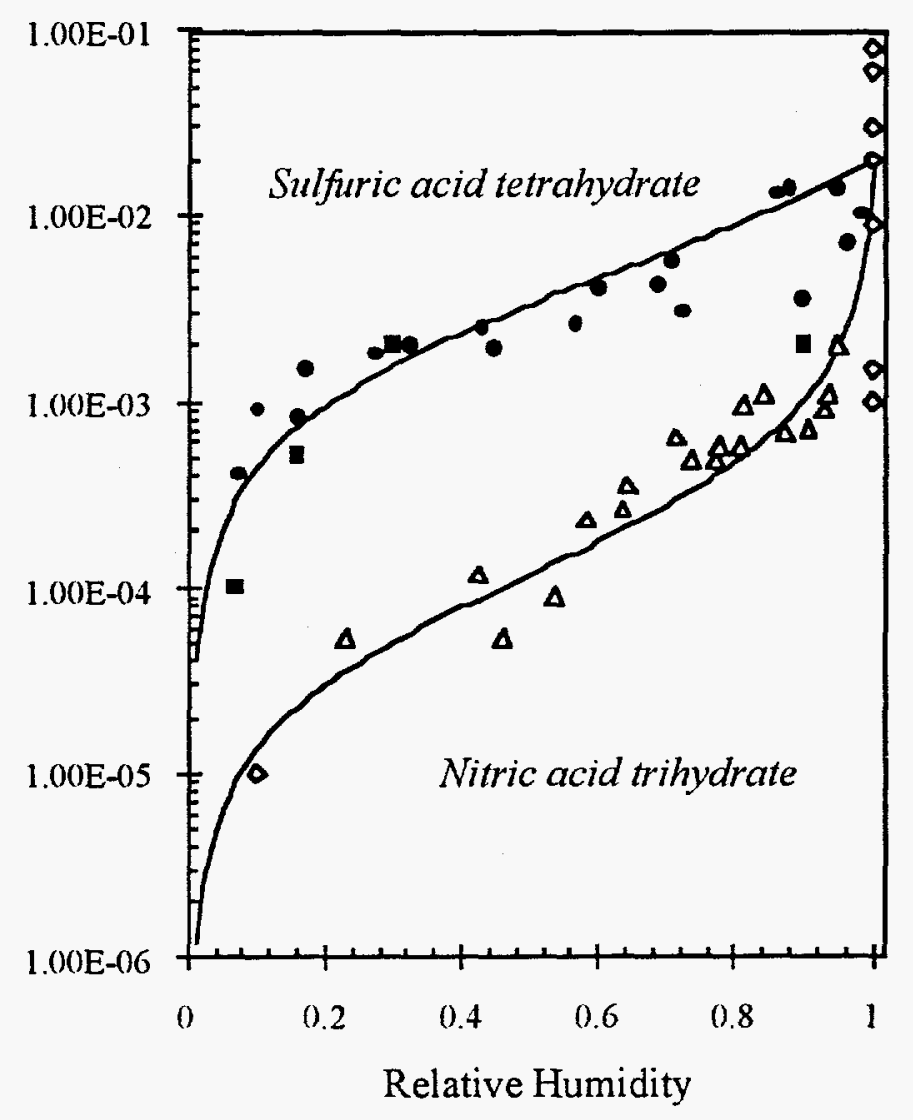

Figure 2. Data for the reaction probability, $\gamma$, as a function of relative humidity for the reaction $\mathrm{ClONO}_{2}+\mathrm{H}_{2} \mathrm{O}$. Data and model calculations for reaction on SAT (filled points) and NAT (open points) are labeled. 


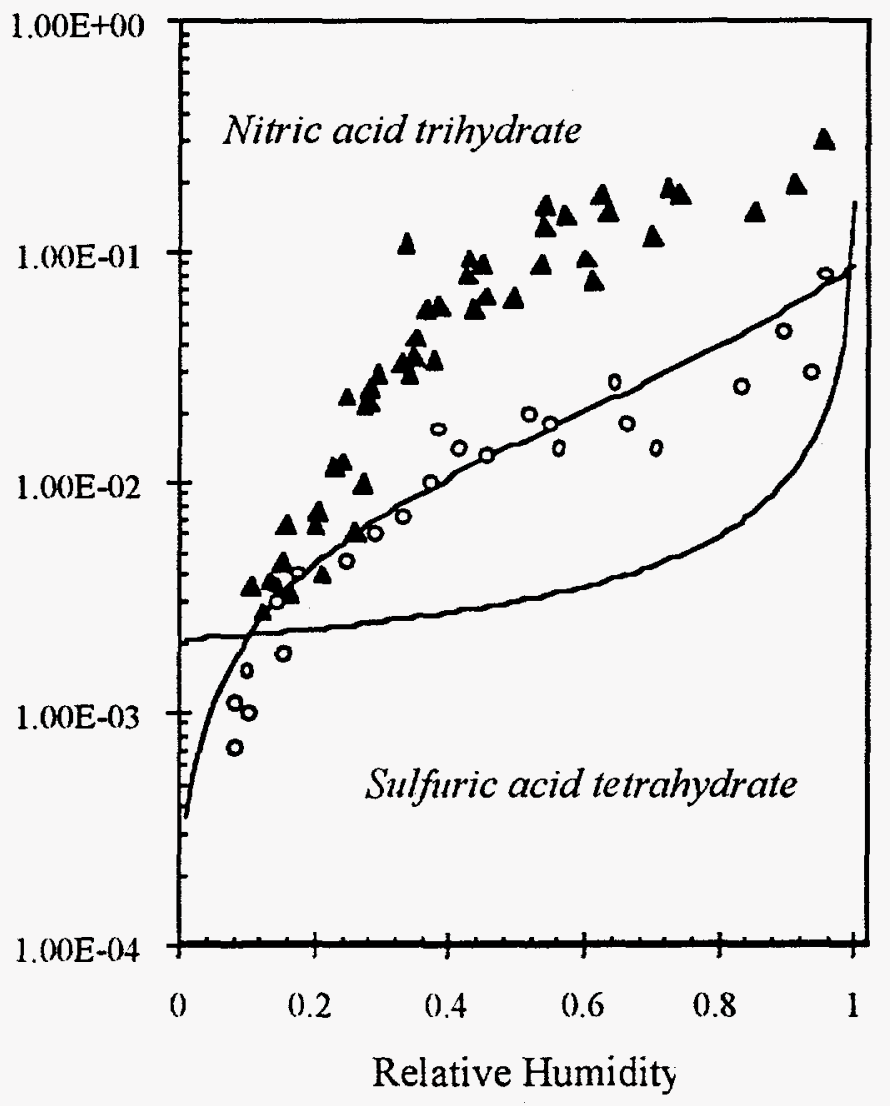

Figure 3. Data for the reaction probability, $\gamma$, as a function of relative humidity for the reaction $\mathrm{ClONO}_{2}+\mathrm{HCl}$. Data and model calculations for reaction on $\mathrm{SAT}_{2}$ (filled points) and NAT (open points) are labeled. 


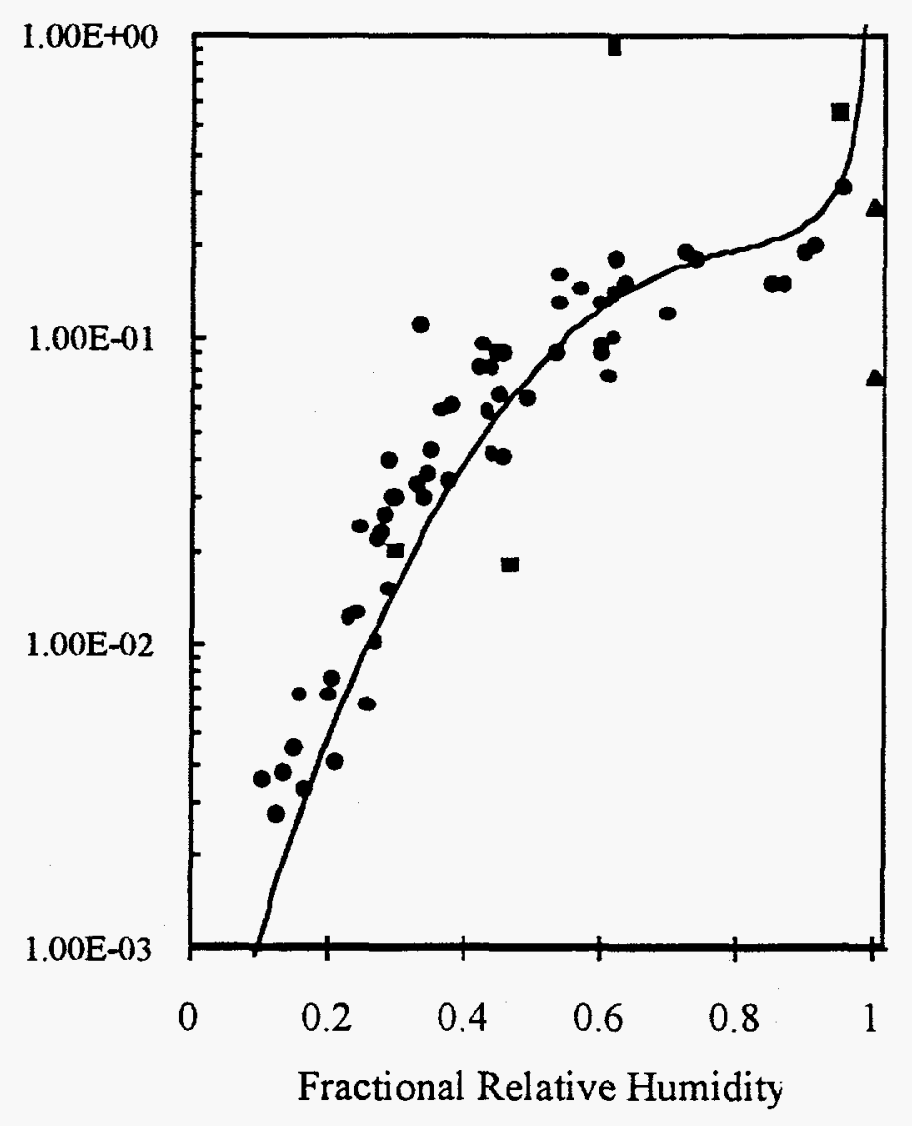

Figure 4. Data for the reaction probability, $\gamma$, as a function of relative humidity for the reaction $\mathrm{ClONO}_{2}+\mathrm{HCl}$ on NAT. Model calculation of reaction probability is based on the capillary absorption mechanism discussed in the text. 